\title{
The Origin of Cultivated Citrus as Inferred from Internal Transcribed Spacer and Chloroplast DNA Sequence and Amplified Fragment Length Polymorphism Fingerprints
}

\author{
Xiaomeng Li and Rangjin Xie \\ College of Horticulture and Landscape Architecture, Southwest University, Chongqing 400716, \\ China; and Citrus Research Institute, Chinese Academy of Agricultural Sciences-Southwest \\ University, Chongqing 400712, China \\ Zhenhua Lu \\ College of Horticulture and Landscape Architecture, Southwest University, Chongqing 400716, \\ China; and Zhengzhou Fruit Research Institute, Chinese Academy of Agricultural Sciences, \\ Zhengzhou 450009, China

\begin{abstract}
Zhiqin Zhou ${ }^{1}$ 400715, China; College of Horticulture and Landscape Architecture, Southwest University, Chongqing 400716, China; and Citrus Research Institute, Chinese Academy of Agricultural Sciences-Southwest University, Chongqing 400712, China
\end{abstract} \\ Key Laboratory of Horticulture for Southern Mountain Regions, Ministry of Education, Chongqing
}

\begin{abstract}
AdDitional INDEX wORDs. phylogeny, maximum parsimony, neighbor-joining, hybrid, parent
Abstract. Citrus species are among the most important fruit trees in the world and have a long cultivation history. However, until now, the exact genetic origins of cultivated Citrus such as sweet orange (Citrus sinensis), lemon (C. limon), and grapefruit ( $C$. paradisi) have remained unidentified. In the present study, amplified fragment length polymorphism (AFLP) fingerprints, nuclear internal transcribed spacer (ITS), and three plastid DNA regions ( $p s b H$ $\operatorname{pet} \mathrm{B}, \operatorname{trn} \mathrm{L}-\operatorname{trn} \mathrm{F}$, and $\operatorname{trn} \mathrm{S}-\operatorname{trn} \mathrm{G})$ of 30 accessions of the cultivated citrus and their putative wild ancestors were analyzed in an attempt to identify their paternal and maternal origins. Molecular phylogenetic trees were constructed based on the AFLP data, and chloroplast DNA and ITS sequences using the genus Poncirus as the outgroup. Our results indicated that bergamot $(C$. aurantifolia) and lemon were derived from citron $(C$. medica) and sour orange $(C$. aurantium), and grapefruit was a hybrid that originated from a cross between pummelo $(C$. grandis) and sweet orange. Rough lemon $(C$. limon) was probable as a parent of rangpur lime $(C$. limonia) and guangxi local lemon $(C$. limonia). Our data also demonstrated that sweet orange and sour orange were hybrids of mandarin $(C$. reticulata) and pummelo, while rough lemon was a cross between citron and mandarin. For mexican lime ( $C$. aurantifolia), our molecular data confirmed a species of Papeda to be the female parent and $C$. medica as the male. These findings provide new information for future study on the taxonomy, evolution, and genetic breeding of Citrus.
\end{abstract}

Citrus species are among the most important fruit crops in the world. They are widely grown in the tropical, subtropical, and borderline subtropical/temperate areas of the world. The genus Citrus was established by Carl Linneaus in 1753 (Swingle and Reece, 1967). Since the establishment of the genus, Citrus has attracted much research interest for more than 250 years (Bayer et al., 2009; Nicolosi et al., 2000; Swingle and Reece, 1967; Tanaka, 1977). The genus Citrus has been variously described as consisting of 1 to 162 species (Malik, 1973; Tanaka,

Received for publication 1 Apr. 2010. Accepted for publication 4 May 2010. This study was supported by the National Natural Science Foundation of China (NSFC grant no. 30671450).

We thank Timothy Schubert (Florida Department of Agriculture and Consumer Service, Division of Plant Industry) and Robert R. Krueger (USDA-ARS, National Clonal Germplasm Repository for Citrus and Dates) for supplying leaf materials. We are grateful to Prof. Daming Zhang from the Institute of Botany, Chinese Academy of Sciences, for providing PCR amplification primers. We would also like to thank Prof. Guangyan Zhong and Mr. Dong Jiang for their help with the experiments.

${ }^{1}$ Corresponding author. E-mail: zqzhoubj@yahoo.com.
1977). The most widely accepted taxonomic systems today are those of Swingle (1943) and Tanaka (1977) who recognized 16 and 162 species, respectively. Scora (1975) and Barrett and Rhodes (1976) suggested that there were only three true species within the subgenus Eucitrus of Swingle's system (i.e., C. medica, C. reticulata, and C. grandis). The other genotypes were derived from hybridization between these true species.

Citrus has a long history of cultivation-more than 4000 years (Scora, 1988; Webber et al., 1967). However, the huge controversy over the phylogeny of key wild species, and the genetic relationship between the cultivated species and their putative wild progenitors have remained unresolved, mainly due to the sexual compatibility between Citrus and related genera, the high frequency of bud mutations, the long history of cultivation, and wide dispersion. Previous studies on the genetic origin of cultivated citrus were mainly based on morphological, biochemical, and isozyme data (Barrett and Rhodes, 1976; Fang, 1993; Scora, 1988; Torres et al., 1978). Recently, DNA markers such as restriction fragment length polymorphisms (RFLPs), random amplified polymorphic DNA 
(RAPD), and sequence-characterized amplified regions (SCARs) were widely used to investigate the phylogenetic relationship of Citrus and its close relatives to infer into the genetic origins of the cultivated types (Federici et al., 1998; Nicolosi et al., 2000). More recently, DNA sequence data gave some clues about the progenitors of cultivated citrus (Bayer et al., 2009). Although these studies provided useful information for the origin of citrus and its taxonomy, the results have not been always in agreement. A clearer understanding of the citrus genetic background is necessary for better a characterization and utilization of citrus germplasm.

Amplified fragment length polymorphism is a whole-genome approach to studying genetic variation at lower-level systematics (Bussell et al., 2005). The AFLP technique has been successfully applied in phylogenetic studies with many important crops such as wheat [Triticum aestivum (Heun et al., 1997)] as well as fruit tree species (Coart et al., 2003; Pang et al., 2007). In particular, the AFLP technique has been used successfully to assess the origin of landrace cultivars of cultivated potato [Solanum tuberosum (Spooner et al., 2005)]. Chloroplast DNA (cpDNA) sequence analysis is especially useful in plant phylogenetic study because of its evolutionary conservatism, the relative abundance in plant tissue, small size (135-160 kb), and predominant uniparental inheritance (Olmstead and Palmer, 1994). The utility of cpDNA to examine maternal lineages of hybrids has been documented in Hippophae goniocarpa (Wang et al., 2008). ITS sequences are biparentally inherited in most angiosperms (Álvarez and Wendel, 2003; Baldwin, 1992). Because of high-frequency gene conversion, conserved size, and great variation, ITS sequences were widely used in phylogenetic tree construction for inferring into the hybrid origin of species (Sang et al., 1995; Siripun and Schilling, 2006).

In the present study, we analyzed three plastid genome regions ( $p s b \mathrm{H}-$ pet $\mathrm{B}, \operatorname{trn} \mathrm{L}-\operatorname{trn} \mathrm{F}$, and $\operatorname{trn} \mathrm{S}-\operatorname{trn} \mathrm{G})$, nuclear ITS sequences, and AFLP fingerprints of 30 citrus accessions in an attempt to construct the cytoplasmic phylogeny of the cultivated citrus and infer into their maternal origins using the cpDNA sequence data, and to reconstruct the nuclear phylogeny of the cultivated citrus and infer into their paternal origins using AFLP and nuclear ITS data. The combination of nuclear DNA and cpDNA data allowed us to identify the exact genetic origin of the cultivated citrus.

\section{Materials and Methods}

Materials. A total of 30 accessions was analyzed in the present study, including 24 belonging to the subgenus Citrus, three from the subgenus Papeda, and three from the genus Poncirus that were used as the outgroups. Detailed information on the materials studied is provided in Table 1.

DNA isolation, AMPlification, AND SEQUENCing. Total DNA was isolated from silica gel-dried leaves following the cetyltrimethylammonium bromide (CTAB) protocol of Doyle and Doyle (1987). The chloroplast spacer regions were amplified using primers $7 \mathrm{U}$ (5'-GGCTACACAAACCGTTGAAG-3') and 7L (5'-ATGTTGACATGAGGAGGAAC-3') for $p s b \mathrm{H}-$ pet $\mathrm{B}$ intergenic spacer (Reverdatto et al., 1989), trnLc (5' CGAAATCGGTAGACGCTACG-3') and trnLf (5'-ATTT GAACTGGTGACACGAG-3') for the $\operatorname{trn} \mathrm{L}$ intron and $\operatorname{trn} \mathrm{L}-$ trnF intergenic spacer (Taberlet et al., 1991), and trnS (GCU) (5'-GAACGAATCACACTTTTACCAC-3') and trnG (UCC)
(5' -GCCGCTTTAGTCCTCAGC-3') for the trnS - trnG intergenic spacer (Hamilton, 1999). The polymerase chain reaction (PCR) reactions were carried out in a total volume of $20 \mu \mathrm{L}$ containing 2.5 to $5 \mathrm{ng}$ of DNA template, $0.3 \mu \mathrm{mol} \cdot \mathrm{L}^{-1}$ of each primer, $0.2 \mathrm{mmol} \cdot \mathrm{L}^{-1}$ of each $\mathrm{dNTP}, 2 \mathrm{mmol} \cdot \mathrm{L}^{-1} \mathrm{MgCl}_{2}, 0.65 \mathrm{U}$ of EX Taq DNA polymerase, and 10×Taq polymerase buffer (TaKaRa Biotechnology, Shiga, Japan). Amplifications were conducted in a T3 Thermocycler (Whatman Biometra, Gottingen, Germany). Polymerase chain reaction cycling parameters for the three cpDNA regions $(p s b \mathrm{H}-p e t \mathrm{~B}, \operatorname{trn} \mathrm{L}-\operatorname{trn} \mathrm{F}$, and $\operatorname{trn} \mathrm{S}-$ $\operatorname{trn} \mathrm{G}$ ) were similar, with an initial denaturation for $4 \mathrm{~min}$ at $94^{\circ} \mathrm{C}$, followed by 30 cycles of $50 \mathrm{~s}$ at $94^{\circ} \mathrm{C}, 30 \mathrm{~s}$ at $57^{\circ} \mathrm{C}, 58^{\circ} \mathrm{C}, 59^{\circ} \mathrm{C}$ for $p s b \mathrm{H}-\operatorname{pet} \mathrm{B}, \operatorname{trn} \mathrm{L}-\operatorname{trn} \mathrm{F}$, and $\operatorname{trn} \mathrm{S}-\operatorname{trn} \mathrm{G}$, respectively, and $1.5 \mathrm{~min}$ at $72{ }^{\circ} \mathrm{C}$, with a final extension time of $10 \mathrm{~min}$ at $72{ }^{\circ} \mathrm{C}$. PCR products were electrophoresed on and excised from a $1.5 \%$ agarose gel, then purified using DNA Fragment Quick Purification / Recover kit (Dingguo Biotechnology, Beijing, China). Sequencing was done on an ABI 3730 (Applied BioSystems, Foster City, CA) by Invitrogen (Invitrogen, Carlsbad, CA).

The ITS fragments including ITS1, ITS2 and 5.8S were amplified with primers AB101 (5'-ACGAATT CATGGTCCGGTGAAGTGTTCG-3') and AB102 (5' TAGAATTCCCCGGTTCG CTCGCCGTTAC-3') (Robinson et al., 2001). The PCR reaction was carried out in a total volume of $25 \mu \mathrm{L}$ containing 5 to $50 \mathrm{ng}$ of template DNA, $0.15 \mu \mathrm{mol} \cdot \mathrm{L}^{-1}$ of each primer, $0.16 \mathrm{mmol} \cdot \mathrm{L}^{-1}$ of each dNTP, $1.5 \mathrm{mmol} \cdot \mathrm{L}^{-1}$ $\mathrm{MgCl}_{2}, 0.75 \mathrm{U}$ of Taq DNA polymerase and $10 \times$ Taq polymerase buffer (TakaRa). Amplification was performed in a T3 Thermocycler (Whatman Biometra) using the following protocol: $2 \mathrm{~min}$ at $96^{\circ} \mathrm{C}$, followed by 30 cycles of $1 \mathrm{~min}$ at $96^{\circ} \mathrm{C}, 1 \mathrm{~min}$ at $60^{\circ} \mathrm{C}$ and $2 \mathrm{~min}$ at $72^{\circ} \mathrm{C}$, and finally $10 \mathrm{~min}$ at $72^{\circ} \mathrm{C}$. Polymerase chain reaction products were electrophoresed on $1.5 \%$ agarose gels and were purified using a DNA Fragment Quick Purification/ Recover kit (Dingguo). The purified ITS templates were ligated to the pGEM-T Easy vector (Promega, Madison, WI) following the manufacturer's protocol and were subsequently transformed into competent cells. At least 14 clones with correct inserts (determined through amplification of PCR products from an entire ITS of each individual) were screened for different ITS types using restriction enzymes EcoRII (/CCWGG), AccBSI (GAG/CGG), AsuC2I (CC/SGG), and MluI (A/CGCGT), which could detect species-specific ITS types among the three true species. Selected positive clones were sequenced in both directions using T7 and SP6 plasmid primers. Sequencing was done on an ABI 3730 (Applied Biosystems) by Invitrogen.

AFLP FINGERPRINTs. The AFLP analyses were performed according to Vos et al. (1995). Genomic DNA ( $\approx 500 \mathrm{ng})$ was digested with restriction enzymes MseI and EcoRI (Fermentas Canada, Burlington, ON, Canada). Digestion and ligation was performed in a single reaction for $6 \mathrm{~h}$ at $37^{\circ} \mathrm{C}$. The preamplification was performed with $M s e I-0$ and EcoRI-0 primer pairs. Selective amplifications were performed with 15 primer combinations (PCs): E-AAC/M-CAA, E-AAC/M-CAC, E-AAC/ M-CAT, E-AAC/M-CTA, E-AAC/M-CTT, E-AAG/M-CAC, E-ACT/M-CTT, E-ACG/M-CTA, E-AGC/M-CA A, E-AGC/ M-CAC, E-AGC/M-CAG, E-AGC/M-CTA, E-AGC/M-CTC, E-AGG/M-CAA, and E-AGG/M-CTG. Selective amplification products were run in a $6 \%$ denaturing polyacrylamide gel. Fragments were scored manually as present (1) or absent (0) in the readable region of bands from 50 to $600 \mathrm{bp}$ in length and were used to construct a binary data matrix with individual plants in rows and bands in columns. 
Table 1. Taxonomy and collection sites of materials used in the study of phylogenetic relationships of the cultivated citrus and their putative wild ancestors using ITS and cpDNA sequence and AFLP fingerprints.

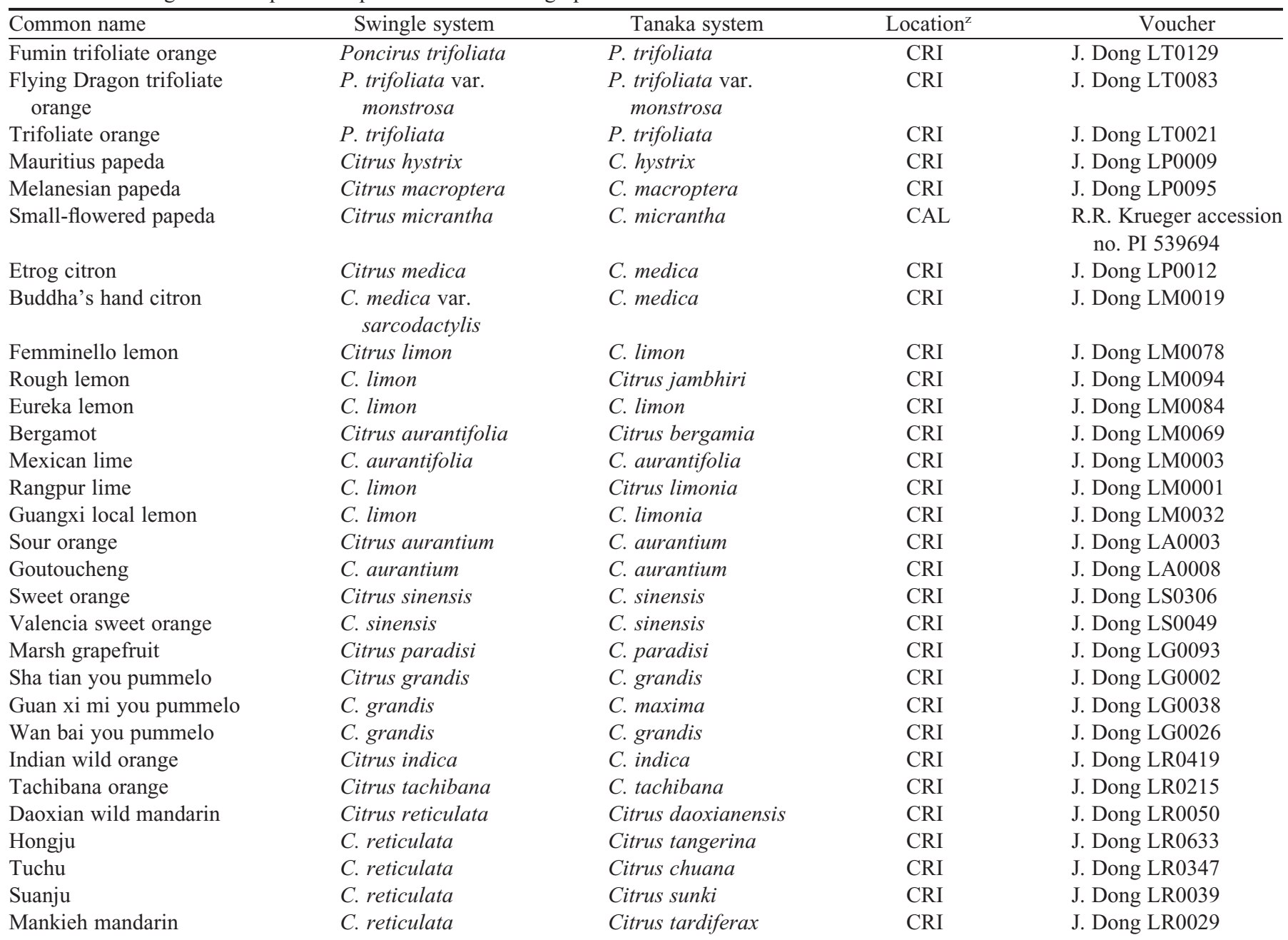

$\overline{{ }^{z} C A L}=$ U.S. Department of Agriculture, Agricultural Research Service, National Clonal Germplasm Repository for Citrus and Dates, Riverside, CA; CRI = Citrus Research Institute, Chinese Academy of Agricultural Sciences, Chongqing, China.

Data analysis. Pairwise genetic distance (D) between all individuals was estimated according Nei and Li's (1979) similarity coefficient and was implemented in the PAUP*, version 4.0b10, software (Swofford, 2000) using the formula: $\mathrm{D}=1-\left(2 \mathrm{n}_{\mathrm{ij}} /\left[\mathrm{n}_{\mathrm{i}}+\mathrm{n}_{\mathrm{j}}\right]\right)$, where $\mathrm{n}_{\mathrm{ij}}$ is the number of shared fragments between two individuals $i$ and $j$, and $n_{i}$ and $n_{j}$ are the total number of fragments in individual $i$ and $j$, respectively. From these values, mean intra- and interspecific distance values were obtained. The cpDNA and ITS sequence data were aligned using ClustalX, version 1.81 (Thompson et al., 1997) with manual adjustments as necessary. Boundaries of $p s b \mathrm{H}-$ pet $\mathrm{B}$, $\operatorname{trn} \mathrm{L}-\operatorname{trn} \mathrm{F}$, and $\operatorname{trn} \mathrm{S}-\operatorname{trn} \mathrm{G}$ regions were determined by comparison with the $C$. sinensis cpDNA sequence (GenBank no. DQ864733). Boundaries of the ITS1 and ITS2 regions and the coding sequences of 5.8S rDNA were determined using available ITS sequence from $C$. maxima (C. grandis; GenBank no. AM398229). The MEGA program, version 3.0 (Kumar et al., 2004), was employed to estimate GC content and nucleotide substitution. The three cpDNA fragments were combined in the maximum parsimony (MP) and neighborjoining (NJ) analyses. The MP and NJ analyses were conducted using computer software PAUP*, version 4.0b10 (Swofford,
2000). All analyses were performed with uninformative characters excluded. In the MP analysis, all characters were assigned equal weight, gaps were treated as missing data, and character states were treated as unordered. A heuristic search was performed with the MULPARS option, tree bisectionreconnection (TBR) branch swapping, and random stepwise addition with 1000 replicates. Topological robustness was assessed by bootstrap analysis with 1000 replicates using simple taxon addition (Felsenstein, 1985).

\section{Results}

Phylogenetic analysis of Chloroplast sequence. The sequences of $p s b \mathrm{H}-\operatorname{pet} \mathrm{B}$ varied from 1089 to $1111 \mathrm{bp}$ in length. The final alignment of $p s b \mathrm{H}-$ pet $\mathrm{B}$ was $1111 \mathrm{bp}$, among which 19 sites were variable and 19 sites were parsimony informative. The sequences of $\operatorname{trn} \mathrm{L}-\operatorname{trnF}$ varied from 967 to $979 \mathrm{bp}$ in length. The final alignment of $\operatorname{trn} \mathrm{L}-\operatorname{trnF}$ was $979 \mathrm{bp}$, and 13 sites were variable and 11 sites were parsimony informative. The amplified $\operatorname{trn} \mathrm{S}-\operatorname{trn} \mathrm{G}$ region length varied from 577 to $647 \mathrm{bp}$, and the final alignment of trnS - trn G was $649 \mathrm{bp}$, including 17 variable sites and 16 informative sites. 
Among the three regions, the informative site of $t r n \mathrm{~S}-\operatorname{trn} \mathrm{G}$ was the highest $(2.47 \%), p s b \mathrm{H}-\operatorname{pet} \mathrm{B}$ was $1.71 \%$, and $\operatorname{trn} \mathrm{L}-\operatorname{trn} \mathrm{F}$ was the lowest $(1.12 \%)$. Nucleotide sequences from the three noncoding regions, $p s b \mathrm{H}-$ pet $\mathrm{B}, \operatorname{trn} \mathrm{L}-t r n \mathrm{~F}$, and $\operatorname{trn} \mathrm{S}-\operatorname{trn} \mathrm{G}$ were combined for this analysis. The combined data set consisted of 2739 characters, of which 2689 sites were constant, and 46 variable characters were phylogenetically informative.

Parsimony analysis of the chloroplast data resulted in a single most parsimonious tree of length 51 steps [Fig. 1; consistency index $(\mathrm{CI})=$ 0.980 , retention index $(\mathrm{RI})=0.995]$. The topologies of the phylogenetic trees based on MP and NJ (data not shown) analyses were identical, differing in statistic support values. From Fig. 1, we can see that the genus Citrus formed a strongly supported monophyletic group (bootstrap support [BS] 100\%). Within the group, there were two major clades, designated A and B. Clade A comprised all accessions of $C$. medica, $C$. reticulata, two accessions of C. limonia, and C. jambhiri (BS 65). Clade B consisted of all accessions of Papeda, C. grandis, $C$. sinensis, $C$. aurantium, and $C$. paradisi 'Marsh', plus two accessions of $C$. limon and $C$. aurantifolia (BS 95). Within clade A, two accessions of $C$. medica formed a strongly supported monophyletic group (BS 100), and C. jambhiri, C. limonia 'Guangxi', and C. limonia 'Rangpur' nested in the mandarin group, forming a strongly supported monophyletic group (BS 99). Within the latter group, C. jambhiri had a close relationship with $C$. limonia 'Rangpur', C. reticulata 'Hongju', and C. reticulata 'Mankieh' (BS 64), while $C$. limonia 'Guangxi', $C$. reticulata 'Daoxian', and $C$. reticulata 'Suanju' grouped together (BS 63). Clade B branched into two subclades, with the first subclade containing three accessions of Papeda and C. aurantifolia 'Mexican' (BS 100), which was sister to the second subclade containing all accessions of C. grandis, C. sinensis, C. aurantium, C. paradisi 'Marsh', and two accessions of $C$. limon and $C$. aurantifolia (BS 95). In the second subclade, there was a polytomy of seven minor clades: C. limon 'Femminello', C. limon 'Eureka', C. bergamia, and $C$. aurantium 'Daidai' formed a clade (BS 64), two accessions of $C$. sinensis were found in another clade (BS 64), and the remaining five accessions separately formed a clade.
Cluster analysis of AFLP data. Initially, 64 AFLP primer combinations were screened while 15 displayed clear stable polymorphisms. The 15 primer combinations generated a total of 233 fragments in the materials studied, of which $187(80.3 \%)$ were polymorphic. On average, 15.5 bands were amplified with one primer combination, of which 12.5 were polymorphic (Table 2). Intra- and interspecific values derived from AFLP data are presented in Table 3. When interspecific mean distances within genus Citrus were compared, $C$. medica and $C$. paradisi appeared to be the most distant pair $(0.0592$, Table 3$)$, while the least 
interspecific distance was between $C$. sinensis and $C$. paradisi (0.0108, Table 3). When comparing intraspecific mean distances, $C$. sinensis appeared to be the least distant $(0.0024$, Table 3).

Of the 233 binary characters, 186 were parsimony informative. A heuristic search resulted in 19 most parsimonious trees with a length of $539(\mathrm{CI}=0.345, \mathrm{RI}=0.674)$ (data not shown). The tree constructed by the NJ analysis is shown in Fig. 2. The topologies of the trees constructed by MP and NJ methods were virtually in congruence. The phylogenetic trees based on both methods strongly supported delineation of the genus Citrus as a monophyletic group (BS 100). The ingroup consisted of a ladder of four clades (designated $\mathrm{C}-\mathrm{F}$ ). Clade $\mathrm{C}$, containing $C$. hystrix, C. macropetera, and C. micrantha was strongly supported (BS 98). Clade D was made up of three accessions of $C$. grandis (BS 74). Clade E consisted of all accessions of C. medica, C. limon, C. aurantifolia, and C. limonia (BS 67). Clade F composed of all accessions of $C$. reticulata, $C$. sinensis, $C$. aurantium, and $C$. paradisi 'Marsh' (with less than $50 \%$ bootstrap support). Within clade $\mathrm{E}$, two accessions of $C$. medica clustered together with $C$. aurantifolia 'Mexican' (BS 66), which all together grouped with the clade containing $C$. limon 'Femminello' and C. limon 'Eureka'. C. jambhiri and $C$. limonia 'Rangpur' formed a strongly supported clade (BS

Table 2. AFLP polymorphism among 30 genotypes of Citrus and Poncirus generated with 15 primer combinations.

\begin{tabular}{llcc}
\hline Primer combinations & $\begin{array}{c}\text { Fragments } \\
\text { (no.) }\end{array}$ & $\begin{array}{c}\text { Polymorphic } \\
\text { fragments (no.) }\end{array}$ & $\begin{array}{c}\text { Polymorphism } \\
\text { rate (\%) }\end{array}$ \\
\hline E-AAC/ M-CAA & 18 & 16 & 88.9 \\
E-AAC/ M-CAC & 14 & 13 & 92.9 \\
E-AAC/ M-CAT & 7 & 4 & 57.1 \\
E-AAC/ M-CTA & 13 & 12 & 92.3 \\
E-AAC/ M-CTT & 17 & 14 & 82.4 \\
E-AAG/ M-CAC & 9 & 7 & 77.8 \\
E-ACT/ M-CTT & 17 & 14 & 82.4 \\
E-ACG/ M-CTA & 23 & 20 & 87.0 \\
E-AGC/ M-CAA & 17 & 14 & 82.4 \\
E-AGC/ M-CAC & 17 & 12 & 70.6 \\
E-AGC/ M-CAG & 17 & 13 & 76.5 \\
E-AGC/ M-CTA & 19 & 15 & 78.9 \\
E-AGC/ M-CTC & 15 & 11 & 73.3 \\
E-AGG/ M-CAA & 20 & 15 & 75.0 \\
E-AGG/ M-CTG & 10 & 7 & 70.0 \\
Sum/mean & $233 / 15.5$ & $187 / 12.5$ & NA/80.3 \\
\hline
\end{tabular}

97), which clade was sister to C. limonia 'Guangxi'. Clade F divided into two subclades (with less than $50 \%$ bootstrap support), with seven accessions of $C$. reticulata forming a strongly supported subclade (BS 83), which became sister to the subclade including all accessions of $C$. sinensis, $C$. aurantium, and $C$. paradisi 'Marsh'. Within the latter subclade, $C$. paradisi 'Marsh' was close to $C$. sinensis (with less than 50\% bootstrap support).

ITS PHYLOGENY. Length variation for the entire ITS region (including the 5.8S coding region) ranged from 642 to $676 \mathrm{bp}$. The ITS1 region ranged from 227 to $257 \mathrm{bp}$, and the ITS2 region ranged from 251 to $256 \mathrm{bp}$. As expected, the 5.8S sequence was identical in size (164 bp) in all the taxa, and no gaps were found when aligning the sequences. The $\mathrm{G}+\mathrm{C}$ content of the sequences ranged from $63.7 \%$ to $67.2 \%$. Aligned ITS sequences were 679 bp long and 614 sites were constant, while 44 of the variable characters were phylogenetically informative. Two species of Poncirus had a unique 4-bp insertion in ITS2. There was a 29-bp deletion in ITS1 of $C$. medica and its putative hybrids. Twelve cultivated accessions, including sour orange, sweet orange, grapefruit, lemon, and lime, had at least two major different ITS types.

Parsimony analysis of the ITS data set resulted in a single most parsimonious tree of length 71 steps $(\mathrm{CI}=0.930, \mathrm{RI}=$ 0.982). The topologies of the phylogenetic trees based on MP and NJ (data not shown) analyses were identical, differing in statistic support values. The most parsimonious tree is shown in Fig. 3. From Fig. 3, it is apparent that the genus Citrus is supported as a monophyletic group (BS 88). The group divided into two large clades (designated $G$ and $H$ ). Clade $G$ was a polytomy containing all accessions of $C$. reticulata and one ITS repeat type of all accessions of $C$. sinensis, $C$. aurantium, C. limon, C. aurantifolia, C. limonia, and C. paradisi 'Marsh', except C. aurantifolia 'Mexican' (BS 91). Clade H consisted of all accessions of Papeda, C. medica, C. grandis, and the other ITS repeat types of all accessions of $C$. sinensis, $C$. aurantium, C. limon, C. aurantifolia, C. limonia, and C. paradisi 'Marsh' (BS 95). Within clade $\mathrm{H}$, there was a polytomy of three minor subclades. The first subclade contained one ITS repeat type of C. limon 'Eureka' and C. aurantium 'Goutoucheng' (BS 100). The second subclade was also a polytomy consisting of three accessions of $C$. grandis, and one ITS repeat type of two accessions of $C$. sinensis, $C$. aurantium, and $C$. paradisi 'Marsh' (BS 95). Within this subclade, C. paradisi 'Marsh' was close to $C$. sinensis (BS 65). The third clade comprised all accessions of Papeda, $C$. medica, and all of the other ITS repeat types of $C$. limon $C$. limonia, and $C$. aurantifolia, except $C$.

Table 3. Intra- and interspecific mean distance values (Nei and Li, 1979) for Citrus taxa based on AFLP data.

\begin{tabular}{|c|c|c|c|c|c|c|c|c|c|c|}
\hline Taxa & Papeda & C. medica & C. limon & C. aurantifolia & C. limonia & C. aurantium & C. sinensis & C. paradisi & C. grandis & C. reticulata \\
\hline Papeda & 0.0222 & & & & & & & & & \\
\hline C. medica & 0.0517 & 0.0055 & & & & & & & & \\
\hline C. limon & 0.0494 & 0.0288 & 0.0126 & & & & & & & \\
\hline C. limonia & 0.0530 & 0.0335 & 0.0144 & 0.0241 & 0.0109 & & & & & \\
\hline C. aurantium & 0.0433 & 0.0536 & 0.0311 & 0.0326 & 0.0305 & 0.0163 & & & & \\
\hline C. sinensis & 0.0467 & 0.0551 & 0.0343 & 0.0370 & 0.0309 & 0.0159 & 0.0024 & & & \\
\hline C. grandis & 0.0513 & 0.0579 & 0.0533 & 0.0483 & 0.0549 & 0.0356 & 0.0348 & 0.0293 & 0.0247 & \\
\hline C. reticulata & 0.0571 & 0.0567 & 0.0351 & 0.0481 & 0.0288 & 0.0256 & 0.0199 & 0.0276 & 0.0496 & 0.0131 \\
\hline
\end{tabular}




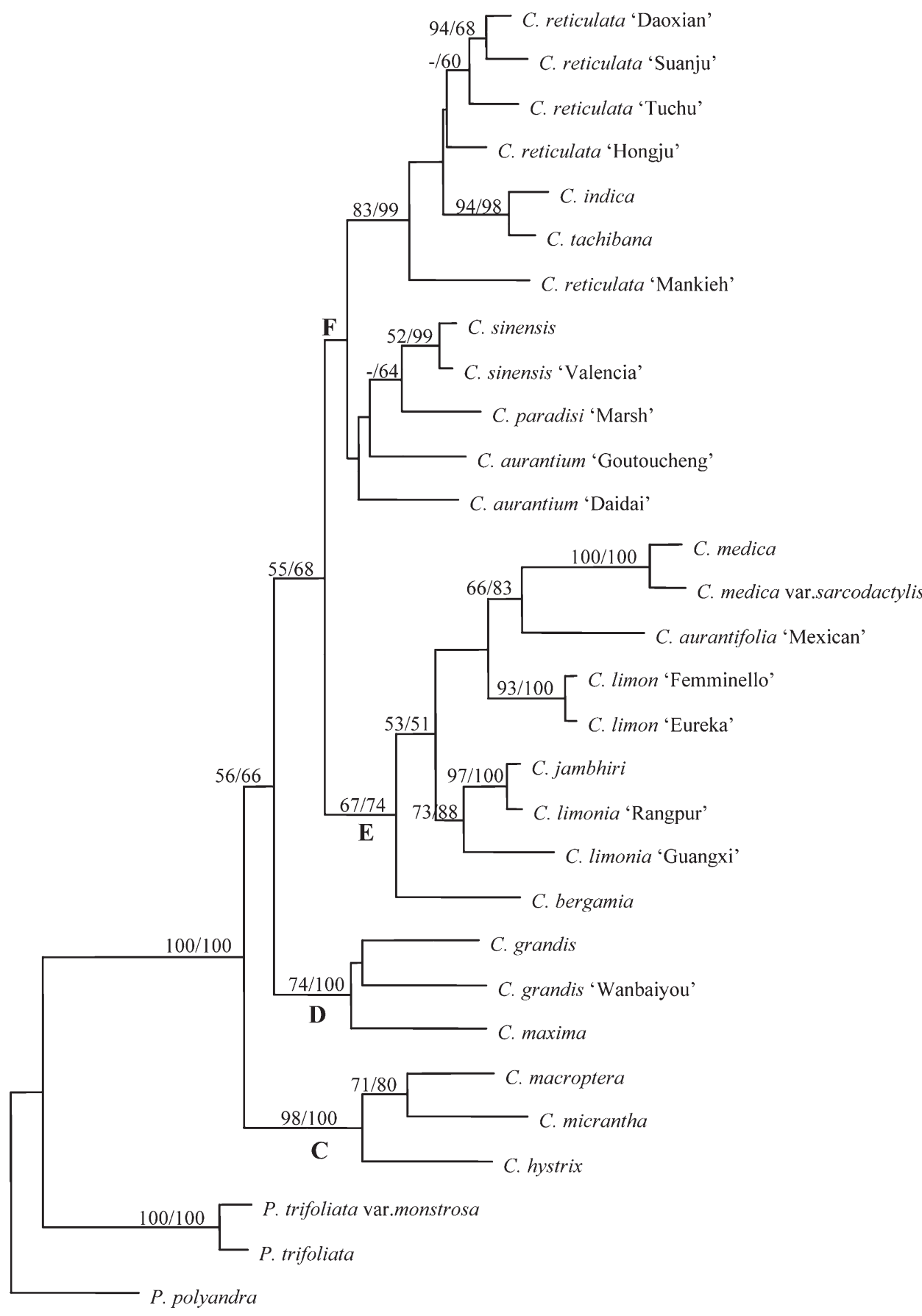

Fig. 2. A neighbor-joining dendrogram for 30 genotypes of Citrus and Poncirus derived from AFLP data. The numbers above branches are bootstrap support percentages higher than $50 \%$ in parsimony and neighbor-joining analyses.

limon 'Eureka'. The third clade (BS 68) branched into two subclades, one being a polytomy containing $C$. hystrix, $C$. macropetera, C. micrantha, and C. aurantifolia 'Mexican' (BS 78). The other was also a polytomy consisting of all accessions of C. medica and one ITS repeat type of C. limon 'Femminello', C. jambhiri, C. bergamia, C. aurantifolia 'Mexican', $C$. limonia 'Rangpur', and C. limonia 'Guangxi' (BS 98).

\section{Discussion}

Because of the widely accepted belief that some or even most Citrus species have been derived from hybridization, in the present study, we used AFLP data to determine paternal origin, cpDNA data to identify maternal origin, and ITS data to assess the full parentage of the cultivated citrus. A combination of nuclear and chloroplast genome information permitted us to make a more concrete conclusion about the exact genetic origin of the cultivated citrus. In the following discussion, we discuss the origins of 12 cultivated citrus types based on previous findings in combination with the new data generated in this study.

SweEt ORANGe (C. SINENSIS). Sweet orange is one of the most widely grown and consumed Citrus types. Based on morphological data, Barrett and Rhodes (1976) suggested that sweet orange was a hybrid of pummelo and mandarin. The suggestion was supported by RAPD and SCAR markers (Nicolosi et al., 2000). Protein data also supported that $C$. reticulata was a parent of sweet orange (Handa et al., 1986; Malik et al., 1974). Chloroplast DNA analyses revealed that the chloroplast genome of sweet orange was derived from pummelo (Barkley et al., 2006; Nicolosi et al., 2000). However, Torres et al. (1978) proposed that sweet orange was improbable as an F1 result of a cross between pummelo and mandarin. In the present study, two accessions of C. sinensis grouped in the pummelo cluster with a strong support (BS 100 ) in the cpDNA tree (Fig. 1). This result strongly supported that pummelo was a maternal parent of sweet orange. On the other hand, our AFLP tree (Fig. 2) clearly indicated that sweet orange was most closely related to mandarin paternally. Most importantly, on our ITS tree (Fig. 3), C. sinensis04 and $C$. sinensis 'Valencia'06 grouped in the mandarin cluster (BS 91), while C. sinensis 06 and $C$. sinensis 'Valencia' 03 clustered with pummelo (BS 95). These results for the first time simultaneously revealed the maternal and paternal origins of sweet orange and provided the concrete molecular evidences that sweet orange was a hybrid of pummelo and mandarin, which acted as the female and male parents, respectively.

Lemon (C. LIMON). Lemon is one of the most important cultivated citrus, mostly used for juice or flavoring. Although lemon was accepted as a species by the Swingle and Tanaka systems (Swingle and Reece, 1967; Tanaka, 1977), the hypotheses on the origin of lemon are divergent. Based on morphological characteristics, Swingle (1943) and Malik et al. (1974) considered 


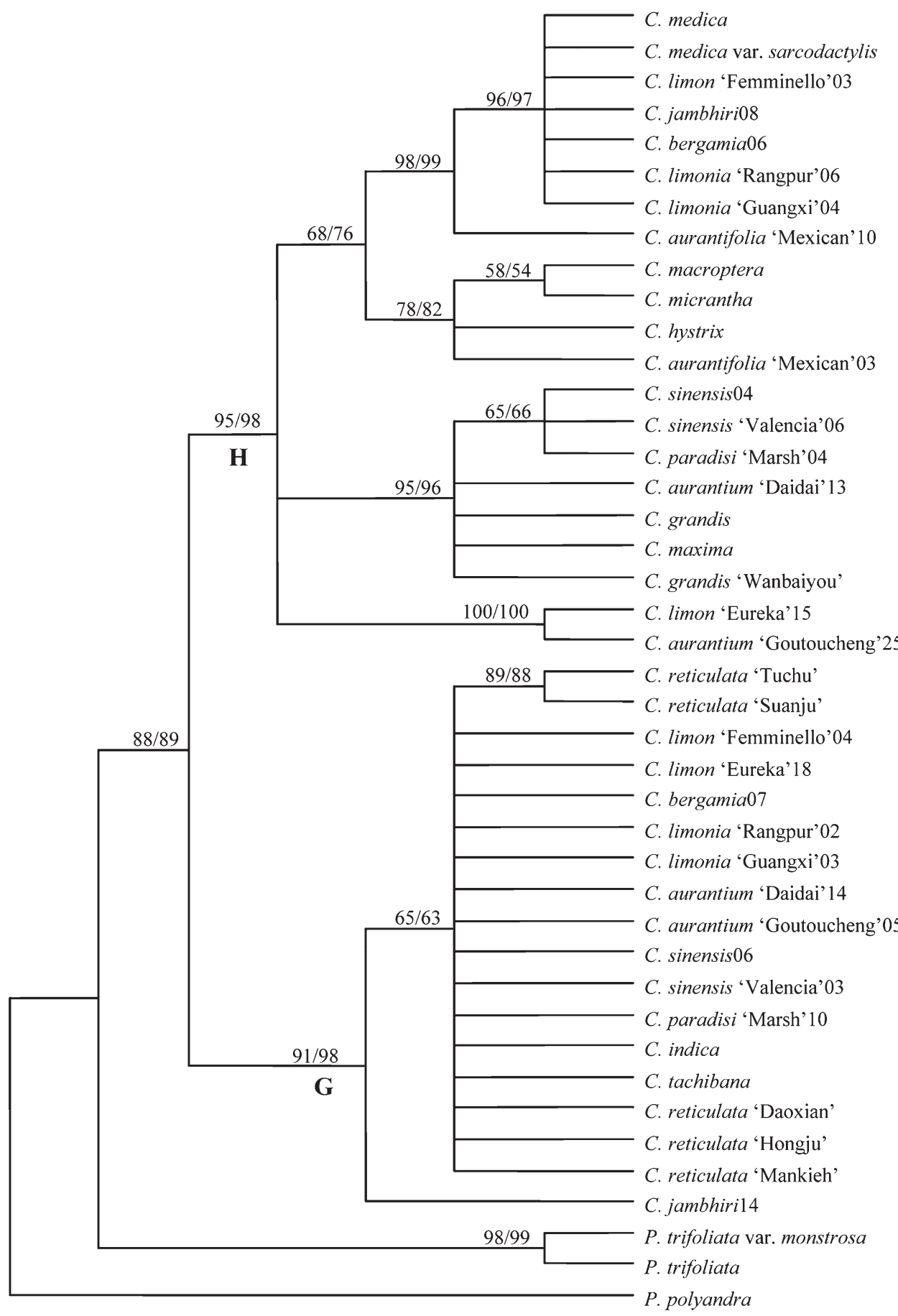

Fig. 3. Single most parsimonious tree for 30 genotypes of Citrus and Poncirus obtained from ITS sequence data (tree length $=71$, consistency index $=0.930$, retention index $=0.982$ ). The numbers above branches are bootstrap support percentages higher than $50 \%$ in parsimony and neighbor-joining analyses. The numbers following the Latin names are the clone numbers.

it to be a hybrid of citron and lime. Barrett and Rhodes (1976) suggested that lemon was a trihybrid of citron, pummelo, and a species of Microcitrus of Tanaka's system. Based on RAPD, SCAR, and cpDNA analysis, Nicolosi et al. (2000) proposed that lemon originated from citron and sour orange, with sour orange being the maternal parent. Citron was a parent of lemon supported by other molecular markers (Barkley et al., 2006; Federici et al., 1998). ISSR data showed that pummelo was a close relative, or a direct or indirect ancestor of lemon (Gulsen and Roose, 2001b). Sour orange as a parent is supported by Hirai and Kozaki (1981) and Torres et al. (1978). In the present study, $C$. limon 'Femminello', C. limon 'Eureka', and C. bergamia clustered with C. aurantium 'Daidai' within the pummelo group (BS 64) based on the cpDNA tree (Fig. 1). This result clearly demonstrated that sour orange was probable a maternal parent of lemon. On the other hand, in our AFLP tree, C. limon 'Femminello', C. limon 'Eureka', and C. jambhiri grouped in the citron cluster (BS 53) (Fig. 2). This result suggested that citron was most likely a parent of lemon. Moreover, on our ITS tree, $C$. jambhiri08 and C. limon 'Femminello'03 grouped in the citron cluster (BS 97), while C. limon 'Eureka'15 clustered with $C$. aurantium 'Goutoucheng'25 (BS 100). These results confirmed that sour orange and citron were parents of lemon. Furthermore, other ITS repeat types of three accessions of lemon (C. jambhiri14, C. limon 'Femminello'04, and C. limon 'Eureka'18) clustered with mandarin (BS 91). This finding suggested that mandarin could be a parent of lemon. However, sour orange was also mandarin hybrid, which could account for the contribution of mandarin gene. Our data provides convincing evidence in supporting the origin of lemon, with sour orange being the maternal parent and citron being the paternal parent.

Webber (1943) thought rough lemon to be a hybrid of lemon and citron. Based on RAPD, RFLP, and SCAR markers, Nicolosi et al. (2000) suggested that citron and mandarin were the ancestors of rough lemon. Citron as a parent of rough lemon was supported by SSR data (Barkley et al., 2006). In the present study, C. jambhiri, C. limonia 'Rangpur', C. reticulata 'Hongju', and C. reticulata 'Mankieh' formed a monophyletic clade (BS 64) in the cpDNA tree (Fig. 1). This result was in agreement with DNA sequence analysis of Bayer et al. (2009), and suggested that mandarin was probably a maternal parent of rough lemon. On the other hand, our AFLP tree showed that C. jambhiri first clustered with $C$. limonia 'Rangpur' and C. limonia 'Guangxi', and then together they grouped with C. limon and C. aurantifolia 'Mexican' in the citron group (BS 53) (Fig. 2). This result indicated that citron was likely to be a paternal parent of rough lemon. In addition, 
two ITS repeat types of rough lemon (C. jambhiri08 and $C$. jambhiri14) grouped in the mandarin and citron clusters with strong support, respectively. These molecular pieces of evidence have more clearly revealed that rough lemon was a hybrid of mandarin and citron, with mandarin being the maternal parent and citron being the paternal parent.

Lemon-LIKe CITRUS (C. LIMONIA). Webber (1943) believed that rangpurs were hybrids of limes and mandarins. The hypothesis was supported by SSR data (Barkley et al., 2006). Nicolosi et al. (2000) suggested that the hybrid origin of rangpur lime was a cross of citron and mandarin hybrid. That mandarin was a parent of rangpur lime was supported by chloroplast data (Gulsen and Roose, 2001a). In the present study, our cpDNA data showed that C. limonia 'Rangpur', $C$. jambhiri, and two accessions of $C$. reticulata formed a monophyletic group within the mandarin group (BS 64), while C. limonia 'Guangxi' clustered with other two accessions of $C$. reticulata (Fig. 1). This result suggested that mandarin was most likely a maternal parent of rangpur lime and guangxi local lemon. On the other hand, our AFLP data demonstrated that $C$. limonia 'Rangpur' clustered together with $C$. jambhiri and C. limonia 'Guangxi' (BS 73) (Fig. 2). This result suggested that rough lemon was probably a paternal parent of rangpur lime and guangxi local lemon. Our results provide additional molecular evidence for the origins of lemon-like citrus. In particular, our ITS analysis demonstrated that $C$. limonia 'Rangpur'02 and C. limonia 'Guangxi'03 clustered in the mandarin group, while $C$. limonia 'Rangpur'06 and C. limonia 'Guangxi'04 grouped in the citron cluster. The finding confirmed the hypothesis that mandarin and citron were parents of lemon-like citrus.

Grapefruit (C. PARADISI). Grapefruit is the one Citrus biotype in which a hybrid origin and subsequent selection for mutants are well documented (Moore, 2001). It was considered to have originated most probably from a hybrid between pummelo and sweet orange (Barrett and Rhodes, 1976), perhaps an introgression back to pummelo (Gmitter, 1995; Moore, 2001). Isozyme data (Fang, 1993; Herrero et al., 1996), SSR data (Barkley et al., 2006), and AFLP data (Pang et al., 2007) clustered grapefruit with pummelo, supporting the suggestion of a backcross with pummelo. However, SCAR (Nicolosi et al., 2000), RFLP plus RAPD (Federici et al., 1998), and other SSR data (Pang et al., 2003), which clustered grapefruit with sweet orange, disagreed with this suggestion. In the present study, C. paradisi 'Marsh' grouped in the pummelo cluster with strong support (BS 100) in the cpDNA tree (Fig. 1). This result suggested that pummelo was most likely a maternal parent of grapefruit. On the other hand, in our AFLP tree, two accessions of $C$. sinensis, $C$. aurantium, and C. paradisi 'Marsh' formed a monophyletic clade with weak support (Fig. 2), and C. paradisi 'Marsh' was closer to $C$. sinensis than $C$. aurantium. This result suggested that sweet orange was possibly a paternal parent of grapefruit. Furthermore, on our ITS tree (Fig. 3), C. paradisi 'Marsh'04, C. sinensis04, and $C$. sinensis 'Valencia'06 clustered together, indicating a close relationship between sweet orange and grapefruit. In particular, C. paradisi 'Marsh'10 grouped in the mandarin cluster (BS 91). This suggested that mandarin could be a parent of grapefruit. However, sweet orange was also a mandarin hybrid, which could account for the contribution of mandarin gene. Our data confirmed that grapefruit was a hybrid of sweet orange and pummelo.
SOUR ORANGE (C. AURANTIUM). Fruits of sour orange are bitter and acidic, thus they were primarily used for their ornamental value and for extracting neroli oil in the past. Because the species is tolerant to cold temperatures and various soil conditions (Castle, 1987), currently, most of them are used principally as a rootstock for sweet orange and other Citrus biotypes. Scora (1975) and Barrett and Rhodes (1976) proposed that sour orange originated from $C$. reticulata and $C$. grandis. The suggestion was supported by isozyme study (Fang, 1993), and RAPD and SCAR markers (Nicolosi et al., 2000). Nicolosi et al. (2000) further revealed that pummelo acted as the female parent of sour orange, which was supported by Barkley et al. (2006) and Bayer et al. (2009). However, Malik et al. (1974) ruled out the possibility that sour orange was a hybrid of sweet orange and mandarin. In the present study, $C$. aurantium 'Daidai' clustered with two accessions of $C$. limon and $C$. bergamia, and then together they formed monophyletic group with three accessions of $C$. grandis, C. aurantium 'Goutoucheng', C. sinensis, and C. paradisi 'Marsh', with strong support (BS 100) in the cpDNA tree (Fig. 1). This result strongly supported that pummelo was probably a maternal parent of sour orange. On the other hand, in our AFLP tree, two accessions of $C$. aurantium and C. sinensis grouped with $C$. paradisi 'Marsh', and then together they formed monophyletic group with all accessions of $C$. reticulata, with weak support (Fig. 2). This result suggested that mandarin was possibly a paternal parent of sour orange. In addition, on our ITS tree (Fig. 3), C. aurantium 'Daidai'14 and C. aurantium 'Goutoucheng'05 grouped in the mandarin cluster (BS 91), while C. aurantium 'Daidai'13 clustered with pummelo (BS 95). Our data confirmed that sour orange was a hybrid of pummelo and mandarin, with pummelo being the maternal parent and mandarin being the paternal parent. In particular, our data have more clearly identified the maternal and paternal origins of sour orange than all other previous data.

Nicolosi et al. (2000) suggested that bergamot was a hybrid of citron and sour orange. That citron was a parent of bergamot was supported by SSR data (Barkley et al., 2006). However, chloroplast data suggested that pummelo was a parent of bergamot (Gulsen and Roose, 2001a; Bayer et al., 2009). In the present study, C. bergamia, C. aurantium 'Daidai', C. limon 'Femminello', and $C$. limon 'Eureka' formed a monophyletic clade (BS 64) in the cpDNA tree (Fig. 1). This result suggested that sour orange was most likely a maternal parent of bergamot. On the other hand, our AFLP tree showed that $C$. bergamia, $C$. limon, $C$. aurantifolia, and $C$. limonia grouped in the citron cluster (BS 67) (Fig. 2). This result indicated that citron was probably a paternal parent of bergamot. In addition, on our ITS tree (Fig. 3), C. bergamia06 grouped in the citron cluster (BS 96), supporting the affinitive relation between bergamot and citron, while $C$. bergamia07 grouped with mandarin (BS 91). This result suggested that mandarin was probable a parent of bergamot. However, sour orange was also mandarin hybrid, which could account for the contribution of the mandarin gene. Our data confirmed that bergamot was a hybrid of sour orange and citron, with sour orange being the maternal parent and citron being the paternal parent.

Lime (C. AURANTIFolia). Barrett and Rhodes (1976) suggested that the lime probably arose from a trihybrid cross involving $C$. medica, $C$. grandis, and a Microcitrus species. Handa et al. (1986) thought that mandarin was a parent of lime, and another parent may be one of citron, pummel, and Papeda. 
Nicolosi et al. (2000) found that all RAPD and SCAR markers present in mexican lime were also present in citron or in $C$. micrantha, suggesting that these two species were the parents. That citron was a parent of limes was supported by isozyme data (Torres et al., 1978) and RFLP data (Federici et al., 1998). Papeda was one parent of lime, supported by SSR data (Barkley et al., 2006). In the present study, C. aurantifolia 'Mexican' clustered with three accessions of Papeda, with strong support (BS 100) in the cpDNA tree (Fig. 1). This result strongly supported that Papeda was most likely a maternal parent of mexican lime. On the other hand, Our AFLP tree indicated that C. aurantifolia 'Mexican' and two accessions of C. medica formed a monophyletic clade (BS 66) (Fig. 2). This result suggested that citron was probable a paternal parent of mexican lime. Meanwhile, on our ITS tree, C. aurantifolia 'Mexican'03 and C. aurantifolia 'Mexican'10 clustered with Papeda and citron groups, respectively. These results strongly supported that mexican lime was a hybrid of Papeda and citron. Our molecular evidence presented more convincing data than all other previous studies in supporting the origin of lime.

\section{Literature Cited}

Álvarez, I. and J.F. Wendel. 2003. Ribosomal ITS sequences and plant phylogenetic inference. Mol. Phylogenet. Evol. 29:417-434.

Baldwin, B.G. 1992. Phylogenetic utility of the internal transcribed spacers of nuclear ribosomal DNA in plants: An example from the compositae. Mol. Phylogenet. Evol. 1:3-16.

Barkley, N.A., M.L. Roose, R.R. Krueger, and C.T. Federici. 2006. Assessing genetic diversity and population structure in a citrus germplasm collection utilizing simple sequence repeat markers (SSRs). Theor. Appl. Genet. 112:1519-1531.

Barrett, H.C. and A.M. Rhodes. 1976. A numerical taxonomic study of affinity relationships in cultivated Citrus and its close relatives. Syst. Bot. 1:105-136.

Bayer, R.J., D.J. Mabberley, C. Morton, C.H. Miller, I.K. Sharma, B.E. Pfeil, S. Rich, R. Hitchcock, and S. Sykes. 2009. A molecular phylogeny of the orange subfamily (Rutaceae: Aurantioideae) using nine cpDNA sequences. Amer. J. Bot. 96:668-685.

Bussell, J.D., M. Waycott, and J.A. Chappill. 2005. Arbitrarily amplified DNA markers as characters for phylogenetic inference. Perspect. Plant Ecol. Evol. Syst. 7:3-26.

Castle, W.S. 1987. Citrus rootstock, p. 361-399. In: R.C. Rom and R.F. Carlson (eds.). Rootstocks for fruit crops. Wiley, New York.

Coart, E., X. Vekemans, M.J.M. Smulders, I. Wagner, J.V. Huylenbroeck, E.V. Bockstaele, and I. Roldán-Ruiz. 2003. Genetic variation in the endangered wild apple (Malus sylvestris (L.) Mill.) in Belgium as revealed by amplified fragment length polymorphism and microsatellite markers. Mol. Ecol. 12:845-857.

Doyle, J.J. and J.L. Doyle. 1987. A rapid DNA isolation procedure for small quantities of fresh leaf tissue. Phytochem. Bul. 19:11-15.

Fang, D.Q. 1993. Intra- and intergeneric relationships of Poncirus polyandar: Investigation by leaf isozymes. J. Wuhan Bot. Res. 11:34-40 (In Chinese with English abstract).

Federici, C.T., D.Q. Fang, R.W. Scora, and M.L. Roose. 1998. Phylogenetic relationships within the genus Citrus (Rutaceae) and related genera as revealed by RFLP and RAPD analysis. Theor. Appl. Genet. 96:812-822.

Felsenstein, J. 1985. Confidence limits on phylogenies: An approach using the bootstrap. Evolution 39:783-791.

Gmitter, F.G. 1995. Origin, evolution and breeding of the grapefruit. Plant Breed. Rev. 13:345-363.

Gulsen, O. and M.L. Roose. 2001a. Chloroplast and nuclear genome analysis of the parentage of lemons. J. Amer. Soc. Hort. Sci. 126: 210-215.
Gulsen, O. and M.L. Roose. 2001b. Lemons: Diversity and relationships with selected Citrus genotypes as measured with nuclear genome markers. J. Amer. Soc. Hort. Sci. 126:309-317.

Hamilton, M.B. 1999. Four primer pairs for the amplification of chloroplast intergenic regions with intraspecific variation. Mol. Ecol. 8:521-523.

Handa, T., Y. Ishizawa, and C. Oogaki. 1986. Phylogenetic study of Fraction I protein in the genus Citrus and its close related genera. Jpn. J. Genet. 61:15-24.

Herrero, R., M.J. Asins, E.A. Carbonell, and L. Navarro. 1996. Genetic diversity in the orange subfamily Aurantioideae. I. Intraspecies and intragenus genetic variability. Theor. Appl. Genet. 92:599-609.

Heun, M., R. Schafer-Pregl, D. Klawan, R. Castagna, M. Accerbi, B. Borghi, and F. Salamini. 1997. Site of einkorn wheat domestication identified by DNA fingerprinting. Science 278:1312-1314.

Hirai, M. and I. Kozaki. 1981. Isozymes of citrus leaves. Proc. Intl. Soc. Citriculture 1:10-13.

Kumar, S., K. Tamura, and M. Nei. 2004. MEGA3: Integrated software for molecular evolutionary genetics analysis and sequence alignment. Brief. Bioinform. 5:150-163.

Malik, M.N. 1973. A new concept in Citrus classification. Pakistan J. Sci. Res. 25:268-271.

Malik, M.N., R.W. Scora, and R.K. Soost. 1974. Studies on the origin of the lemon. Hilgardia 42:361-382.

Moore, G.A. 2001. Oranges and lemons: Clues to the taxonomy of Citrus from molecular markers. Trends Genet. 17:536-540.

Nei, M. and W.H. Li. 1979. A mathematical model for studying genetic variation in terms of restriction endonucleases. Proc. Natl. Acad. Sci. USA 75:5269-5273.

Nicolosi, E., Z.N. Deng, A. Gentile, S. La Malfa, G. Continella, and E. Tribulato. 2000. Citrus phylogeny and genetic origin of important species as investigated by molecular markers. Theor. Appl. Genet. 100:1155-1166.

Olmstead, R.G. and J.D. Palmer. 1994. Chloroplast DNA systematics: A review of method and data analysis. Amer. J. Bot. 81:1205-1224.

Pang, X.M., C.G. Hu, and X.X. Deng. 2003. Phylogenetic relationships among Citrus and its relatives as revealed by SSR markers. Acta Genetica Sinica 30:81-87.

Pang, X.M., C.G. Hu, and X.X. Deng. 2007. Phylogenetic relationships within Citrus and its related genera as inferred from AFLP markers. Genet. Resources Crop Evol. 54:429-436.

Reverdatto, S.V., A.V. Andreeva, A.A. Buryakova, O.G. Chakhmakhcheva, and V.A. Efimov. 1989. Nucleotide sequence of the $5.2 \mathrm{kbp}$ barley chloroplast DNA fragment, containing $p s b \mathrm{~B}-p s b \mathrm{H}-\operatorname{pet} \mathrm{B}-$ pet $\mathrm{D}$ gene cluster. Nucleic Acids Res. 17:2859-2860.

Robinson, J.P., S.A. Harris, and B.E. Juniper. 2001. Taxonomy of the genus Malus Mill. (Rosaceae) with emphasis on the cultivated apple, Malus domestica Borkh. Plant Syst. Evol. 226:35-58.

Sang, T., D.J. Crawford, and T.F. Stuessy. 1995. Documentation of reticulate evolution in peonies (paeonia) using internal transcribed spacer sequences of nuclear ribosomal DNA: Implications for biogeography and concerted evolution. Proc. Natl. Acad. Sci. USA 92: 6813-6817.

Scora, R.W. 1975. On the history and origin of Citrus. Bull. Torrey Bot. Club 102:369-375.

Scora, R.W. 1988. Biochemistry, taxonomy and evolution of modern cultivated Citrus, p. 277-289. In: R. Goren and K. Mendel (eds.). Proc. 6th Intl. Citrus Congr. Vol. 1. Margraf, Weikersheim, Germany.

Siripun, K.C. and E.E. Schilling. 2006. Molecular confirmation of the hybrid origin of Eupatorium godfreyanum (Asteraceae). Amer. J. Bot. 93:319-325.

Spooner, D.M., K. Mclean, G. Ramsay, R. Waugh, and G.J. Bryan. 2005. A single domestication for potato based on multilocus amplified fragment length polymorphism genotyping. Proc. Natl. Acad. Sci. USA 102:14694-14699.

Swingle, W.T. 1943. The botany of Citrus and its wild relatives of the orange subfamily, p. 129-474. In: H.J. Webber and L.D. Batchelor (eds.). The citrus industry. Vol. 1. Univ. of California, Berkeley. 
Swingle, W.T. and P.C. Reece. 1967. The botany of Citrus and its wild relatives, p. 190-423. In: W. Reuther, H.J. Webber, and L.D. Batchelor (eds.). The citrus industry. Vol. 1. Univ. of California, Berkeley.

Swofford, D.L. 2000. PAUP*. Phylogenetic analysis using parsimony (* and other methods). Version 4b. Sinauer, Associates, Sunderland, MA.

Taberlet, P., L. Gielley, G. Pautou, and J. Bouvet. 1991. Universal primers for amplification of three non-coding regions of chloroplast DNA. Plant Mol. Biol. 17:1105-1109.

Tanaka, T. 1977. Fundamental discussion of Citrus classification. Studia Citrologica 14:1-6.

Thompson, J.D., T.J. Gibson, F. Plewniak, F. Jeanmougin, and D.G. Higgins. 1997. The Clustal-X Windows interface: Flexible strategies for multiple sequence alignment aided by quality analysis tools. Nucleic Acids Res. 25:4876-4882.
Torres, A.M., R.K. Soost, and U. Diedenhofen. 1978. Leaf isozymes as genetic markers in Citrus. Amer. J. Bot. 65:869-881.

Vos, P., R. Hogers, M. Bleeker, M. Reijans, T.d. Lee, M. Hornes, A. Frijters, J. Pot, J. Peleman, M. Kuiper, and M. Zabeau. 1995. AFLP: A new technique for DNA fingerprinting. Nucleic Acids Res. 23: 4407-4414.

Wang, A., F. Schluetz, and J. Liu. 2008. Molecular evidence for double maternal origins of the diploid hybrid Hippophae goniocarpa (Elaeagnaceae). Bot. J. Linn. Soc. 156:111-118.

Webber, H.J. 1943. Cultivated varieties of Citrus, p. 475-668. In: H.J. Webber and L.D. Batchelor (eds.). The citrus industry, Vol. I. Univ. of California, Berkeley.

Webber, H.J., W. Reuther, and H.W. Lawton. 1967. History and development of the citrus industry, p. 1-39. In: W. Reuther, H.J. Webber, and L.D. Batchelor (eds.). The citrus industry, Vol. I. Univ. of California, Berkeley. 